\title{
$R$-GROUPS AND ELLIPTIC REPRESENTATIONS FOR UNITARY GROUPS
}

\author{
DAVID GOLDBERG
}

(Communicated by Roe Goodman)

\begin{abstract}
We determine the reducibility and number of components of any representation of a quasi-split unitary group which is parabolically induced from a discrete series representation. The $R$-groups are computed explicitly, in terms of reducibility for maximal parabolics. This gives a description of the elliptic representations.
\end{abstract}

\section{INTRODUCTION}

Let $G$ be a reductive $p$-adic group. That is, $G=\mathbf{G}(F)$, where $\mathbf{G}$ is a reductive algebraic group, defined over a $p$-adic field $F$ of characteristic zero. Let $P=M N$ be a parabolic subgroup of $G$. Suppose $\sigma$ is a discrete series representation of $M$. Consider the unitarily induced representation $i_{G, M}(\sigma)=$ $\operatorname{Ind}_{P}^{G}\left(\sigma \otimes 1_{N}\right)$. We are interested in determining when $i_{G, M}(\sigma)$ is reducible, and if so, what can be said about the constituents. A complete classification of the irreducible constituents of the representations $i_{G, M}(\sigma)$ gives rise to a classification of the tempered spectrum of $G$.

The theory of $R$-groups tells one exactly when $i_{G, M}(\sigma)$ is reducible, and describes the number of irreducible components and their multiplicity. In [4] we explicitly described all the $R$-groups for the split classical groups $S p(2 n)$ and $S O(n)$. In [3] we described the $R$-groups for $S L(n)$. We now explicitly describe the structure of the $R$-groups when $G$ is either of the quasi-split unitary groups, $U(n, n)$ or $U(n, n+1)$. These results are remarkably similar to those for the groups $S p(2 n)$ and $S O(2 n+1)$. Every $R$-group we consider is of the form $\mathbb{Z}_{2}^{d}$, and the integer $d$ can be described explicitly in terms of $\sigma$.

Let $G^{e}$ be the set of regular elliptic elements of $G$. Suppose $\pi$ is an irreducible tempered representation of $G$ with character $\Theta_{\pi}$. Let $\Theta_{\pi}^{e}$ denote the restriction of $\Theta_{\pi}$ to $G^{e}$. Then $\pi$ is said to be elliptic if $\Theta_{\pi}^{e}$ is nonzero. In [1], Arthur gives a characterization of the elliptic constituents of $i_{G, M}(\sigma)$ in terms of $R$-groups. In [7], Herb classifies the elliptic representations of $S p(2 n)$ and $S O(n)$ by using Arthur's description. We describe the elliptic representations of quasi-split unitary groups by following [7]. Again the results are similar to those for the split groups.

Received by the editors February 10, 1992 and, in revised form, July 1, 1993.

1991 Mathematics Subject Classification. Primary 22E50, 22E35.

(C) 1995 American Mathematical Society $0002-9939 / 95 \$ 1.00+\$ .25$ per page 
In $\S 1$ we will review the theory of intertwining operators and $R$-groups. In $\S 2$ we will describe the structure of the parabolic subgroups of $U(n, n)$ and $U(n, n+1)$. In $\S 3$ we compute the $R$-group attached to any discrete series representation of any Levi component of $G$. In $\S 4$ we describe the commuting algebra of $i_{G, M}(\sigma)$ and use this to describe the elliptic representations. As a result of the description of the commuting algebra, we find that every discrete series representation of every Levi component induces with multiplicity 1. Moreover, every irreducible tempered representation is either elliptic or is irreducibly induced from a tempered elliptic representation of a proper parabolic subgroup. This is not the case for every group $G$, as is pointed out in [7]. Further counterexamples can be found in [3].

\section{Preliminaries}

Let $F$ be a locally compact, nondiscrete, nonarchimedean local field of characteristic zero and residual characteristic $q_{F}$. Let $G$ be the $F$-rational points of a connected reductive quasi-split algebraic group defined over $F$. Let $G^{\prime}$ be the set of regular elements of $G$ [6]. We say an element $x$ of $G$ is elliptic if its centralizer is compact, modulo the center of $G$. We denote by $G^{e}$ the set of regular elliptic elements of $G$.

We let $\mathscr{E}_{2}(G)$ denote the collection of equivalence classes of irreducible discrete series representations of $G$. We write $\mathscr{E}_{t}(G)$ for the collection of equivalence classes of irreducible tempered representations of $G$. Then $\mathscr{E}_{2}(G) \subset$ $\mathscr{E}_{t}(G)$. If $\pi \in \mathscr{E}_{t}(G)$, then we write $\boldsymbol{\Theta}_{\pi}$ for the character of $\pi$ and $\boldsymbol{\theta}_{\pi}^{e}$ for its restriction to $G^{e}$. We say that $\pi$ is elliptic if $\theta_{\pi}^{e} \neq 0$.

We say that $M \subseteq G$ is a Levi subgroup of $G$ if there is a parabolic subgroup $P$ of $G$, with $M$ the Levi component of $P$. Let $A_{0}$ be a maximal $F$-split torus of $G$, and let $\Phi\left(G, A_{0}\right)$ be the set of restricted roots of $G$ with respect to $A_{0}$. Let $\Delta$ be a collection of simple roots in $\Phi$. Then the conjugacy classes of parabolic subgroups are in one-to-one correspondence with the subsets of $\Delta$ [16]. If $\theta \subset \Delta$, then we let $A_{\theta} \subset A_{0}$ be the subtorus corresponding to $\theta$. Then $M_{\theta}=Z_{G}\left(A_{\theta}\right)$ is the the Levi subgroup corresponding to $\theta$. We denote by $B=T U$ the Borel subgroup associated to $A_{\varnothing}=A_{0}$.

Let $M$ be a Levi subgroup of $G$. Let $(\sigma, V)$ be a smooth representation of $M$. Suppose $P=M N$ is a parabolic subgroup with Levi component $M$. We let $\operatorname{Ind}_{P}^{G}(\sigma)$ be the representation unitarily induced by $\sigma$. Since the class of $\operatorname{Ind}_{P}^{G}(\sigma)$ depends only on $M$, and not $P$, we also write $i_{G, M}(\sigma)$ for $\operatorname{Ind}_{P}^{G}(\sigma)$.

Let $A=A_{\theta}$ and $M=M_{\theta}$. We denote by $W(A)$ or $W(G / A)$, the Weyl group $N_{G}(A) / M$ of $G$ with respect to $A$. For $w \in W(A)$, we make no distinction between $w$ and a representative for $w$ in $N_{G}(A)$. If $\sigma$ is an irreducible smooth representation of $M$ and $w \in W(A)$, then we let $w \sigma$ be the representation defined by $w \sigma(m)=\sigma\left(w^{-1} m w\right)$. We say that $\sigma$ is ramified if there is some $w \in W(A), w \neq 1$, so that $\sigma \simeq w \sigma$.

We denote by $X(M)_{F}$ the collection of $F$-rational characters of $M$. Then $\mathfrak{a}=\operatorname{Hom}\left(X(M)_{F}, \mathbb{R}\right)$ is the real Lie algebra of $A$, and we let $\mathfrak{a}_{\mathrm{C}}^{*}$ be the complexified dual of $\mathfrak{a}$ [6]. There is a homomorphism $H_{P}: M \rightarrow \mathfrak{a}$ satisfying

$$
q_{F}^{\left\langle\chi, H_{P}(m)\right\rangle}=|\chi(m)|_{F}, \quad \forall \chi \in X(M)_{F}, m \in M .
$$

Let $\nu \in \mathfrak{a}_{\mathrm{C}}^{*}$ and $\sigma \in \mathscr{E}_{2}(M)$. We denote the representation $\operatorname{Ind}_{P}^{G}\left(\sigma \otimes q_{F}^{\left\langle\nu, H_{P}(-)\right\rangle}\right)$ 
by $I(\nu, \sigma)$. Let $w \in W(A)$, and let $N_{w}=U \cap w^{-1} \bar{N} w$, where $\bar{N}$ is the unipotent radical opposed to $N$. Suppose $f$ is a function in the space of $I(\nu, \sigma)$. We formally define an operator by

$$
A(\nu, \sigma, w) f(g)=\int_{N_{w}} f\left(w^{-1} n g\right) d n .
$$

We say $A(\nu, \sigma, w)$ converges if (1.1) converges for each choice of $f$ and $g$. Normalizing by a meromorphic scalar factor we obtain a family of intertwining operators between $I(\nu, \sigma)$ and $I(w \nu, w \sigma)$, which are holomorphic on the unitary axis $i \mathfrak{a}^{*}[14,16]$. We denote these operators by $\mathscr{A}(\nu, \sigma, w)$ and write $\mathscr{A}(\sigma, w)=\mathscr{A}(0, \sigma, w)$. Then these operators satisfy the cocycle condition

$$
\mathscr{A}\left(\sigma, w_{1} w_{2}\right)=\mathscr{A}\left(w_{2} \sigma, w_{1}\right) \mathscr{A}\left(w_{2}, \sigma\right) \text {. }
$$

Theorem 1.1 (Harish-Chandra). Let $w \in W(A)$ and $\sigma \in \mathscr{E}_{2}(M)$. Then there is a complex number $\mu(\nu, \sigma, w)$ so that, if Haar measure is chosen appropriately,

$$
A(\nu, \sigma, w) A\left(w \nu, w \sigma, w^{-1}\right)=\mu(\nu, \sigma, w)^{-1} \gamma_{w}^{2}(G / P),
$$

where $\gamma_{w}(G / P)$ is defined in [6]. Moreover, $\nu \mapsto \mu(\nu, \sigma, w)$ is meromorphic on $a_{\mathfrak{C}}^{*}$, and holomorphic and nonnegative on the unitary axis $i \mathrm{a}^{*}$.

The scalar $\mu(\nu, \sigma, w)$ is called the Plancherel measure attached to $\nu, \sigma$, and $w$. If $w$ is the longest element of the Weyl group, then we write $\mu(\nu, \sigma)$ for $\mu(\nu, \sigma, w)$. We also write $\mu(\sigma)$ for $\mu(0, \sigma)$. If $M$ is maximal and proper, then $i_{G, M}(\sigma)$ is reducible if and only if $\sigma$ is ramified and $\mu(\sigma) \neq 0[16$, Corollary 5.4.2.3].

Let $W(\sigma)=\{w \in W(A) \mid w \sigma \simeq \sigma\}$. Choose an intertwining operator $T(w)$ satisfying $T(w)(w \sigma)=\sigma T(w)$. Then $\mathscr{A}^{\prime}(\sigma, w)=T(w) \mathscr{A}(\sigma, w)$ is a selfintertwining operator for $i_{G, M}(\sigma)$. Let $C(\sigma)$ be the commuting algebra of $i_{G, M}(\sigma)$.

Theorem 1.2 (Harish-Chandra, [16, Theorem 5.5.3.2]). The collection

$$
\left\{\mathscr{A}^{\prime}(\sigma, w) \mid w \in W(\sigma)\right\}
$$

spans the commuting algebra $C(\sigma)$.

Therefore, we would like to be able to determine a basis for $C(\sigma)$ from among the operators $\mathscr{A}^{\prime}(\sigma, w)$. This leads to the construction of the KnappStein $R$-group. Let $\Phi(P, A)$ be the set of reduced roots of $P$ with respect to $A$. Let $\beta \in \Phi(P, A)$, and let $A_{\beta}$ be the torus $(\operatorname{ker} \beta \cap A)^{0}$. Suppose $M_{\beta}$ is the centralizer of $A_{\beta}$ in $G$. Note that $M$ is a maximal proper Levi subgroup of $M_{\beta}$. Let $\mu_{\beta}(\sigma)$ denote the Plancherel measure attached to $i_{M_{\beta}, M}(\sigma)$. Let $\Delta^{\prime}=\left\{\beta \in \Phi(P, A) \mid \mu_{\beta}(\sigma)=0\right\}$. We let $R=\left\{w \in W(\sigma) \mid w \beta>0, \forall \beta \in \Delta^{\prime}\right\}$. Since $\mu_{w \beta}(\sigma)=\mu_{\beta}\left(w^{-1} \sigma\right)$ [6, p. 183], $R=\left\{w \in W(\sigma) \mid w \Delta^{\prime}=\Delta^{\prime}\right\}$. Let $W^{\prime}$ be the subgroup of $W(\sigma)$ generated by the reflections in the roots of $\Delta^{\prime}$.

Theorem 1.3 (Knapp-Stein, Silberger $[11,15])$. For any $\sigma \in \mathscr{E}_{2}(M), W(\sigma)=$ $R \ltimes W^{\prime}$. Furthermore, $W^{\prime}=\left\{w \in W(\sigma) \mid \mathscr{A}^{\prime}(\sigma, w)\right.$ is scalar $\}$.

Therefore, $\left\{\mathscr{A}^{\prime}(\sigma, w) \mid w \in R\right\}$ is a basis for the commuting algebra $C(\sigma)$. However, we can say even more. If $w_{1}, w_{2} \in R$, then

$$
\mathscr{A}^{\prime}\left(\sigma, w_{1} w_{2}\right)=\eta\left(w_{1}, w_{2}\right) \mathscr{A}^{\prime}\left(\sigma, w_{1}\right) \mathscr{A}^{\prime}\left(\sigma, w_{2}\right),
$$


where the 2-cocycle $\eta: R \times R \rightarrow \mathbb{C}^{*}$ satisfies

$$
T\left(w_{1} w_{2}\right)=\eta\left(w_{1}, w_{2}\right) T\left(w_{1}\right) T\left(w_{2}\right) .
$$

Theorem 1.4 (See $[10])$. (a) $C(\sigma) \simeq \mathbb{C}[R]_{\eta}$, where $\mathbb{C}[R]_{\eta}$ is the group algebra twisted by the cocycle $\eta$.

(b) Suppose $R$ is abelian and $\eta$ splits, i.e., $C(\sigma) \simeq \mathbb{C}[R]$. Then $i_{G, M}(\sigma)$ decomposes with multiplicity one.

The number of inequivalent constituents is equal to the number of irreducible representations of $R$, and the irreducible component corresponding to $\rho \in \hat{R}$ appears with multiplicity $\operatorname{dim} \rho$. Let $\mathscr{H}$ be the space of $\operatorname{Ind}_{P}^{G}(\sigma)$. Let $\pi_{\rho}$ be the irreducible constituent corresponding to $\rho$, and let $\theta_{\rho}$ be the character of $\rho$. We define an operator $\mathscr{A}_{\rho}$ by

$$
\mathscr{A}_{\rho}=|R|^{-1} \operatorname{dim} \rho \sum_{w \in R} \bar{\theta}_{\rho}(w) \mathscr{A}^{\prime}(\sigma, w) .
$$

Then $\left\{\mathscr{A}_{\rho} \mid \rho \in \hat{R}\right\}$ is a collection of nonzero [15] orthogonal projections, and $\mathscr{H}_{\rho}=\mathscr{A}_{\rho} \mathscr{H}$ is the $\pi_{\rho}$ isotypic subspace of $\mathscr{H}$. Note that if $R$ is abelian and $\eta$ splits, then $\mathscr{H}_{\rho}=\left\{v \in \mathscr{H} \mid \mathscr{A}^{\prime}(w, \sigma) v=\rho(w) v\right.$ for all $\left.w \in R\right\}$ and $\left(\pi_{\rho}, \mathscr{X}_{\rho}\right)$ is irreducible [10].

We now recall some results of Arthur [1] and Herb [7] on elliptic representations. For each $w \in R$ define $\mathfrak{a}_{w}=\{H \in \mathfrak{a} \mid w \cdot H=H\}$. Let $Z$ be the split component of $G$, i.e., the maximal $F$-split torus in the center of $G$; and let $\mathfrak{z}$ be the real Lie algebra of $Z$. Then $\mathfrak{z} \subset \mathfrak{a}_{w}$ for each $w \in R$. Let $\mathfrak{a}_{R}=\bigcap_{w \in R} \mathfrak{a}_{w}$. We state a weak version of Arthur's theorem. The hypotheses will apply in all of our examples.

Theorem 1.5 (Arthur [1, Proposition 2.1]). Suppose $R$ is abelian and $C(\sigma) \simeq$ $\mathbb{C}[R]$. Then the following are equivalent:

(a) $i_{G, M}(\sigma)$ has an elliptic constituent,

(b) all the constituents of $i_{G, M}(\sigma)$ are elliptic,

(c) there is a $w \in R$ so that $\mathfrak{a}_{w}=\mathfrak{z}$.

For the remainder of this section we suppose $R$ is abelian and $C(\sigma) \simeq \mathbb{C}[R]$. Suppose $M^{\prime} \supset M$ is a Levi subgroup of $G$ satisfying Arthur's compatibility condition with respect to $\Delta^{\prime}[1, \S 2]$. Then $R^{\prime}=R \cap W\left(M^{\prime} / A\right)$ is the $R$ group attached to $i_{M^{\prime}, M}(\sigma)$. If $\kappa^{\prime} \in \hat{R}^{\prime}$, then we let $\tau_{\kappa^{\prime}}$ to be the irreducible component of $i_{M^{\prime}, M}(\sigma)$ corresponding to $\kappa^{\prime}$. Let $\hat{R}\left(\kappa^{\prime}\right)=\{\kappa \in \hat{R} \mid \kappa(w)=$ $\left.\kappa^{\prime}(w), \forall w \in R^{\prime}\right\}$. We let $\pi_{\kappa}$ be the irreducible constituent of $i_{G, M}(\sigma)$ corresponding to $\kappa$.

Theorem 1.6 (Arthur [1, $\S 2]$ ). For any $\kappa^{\prime} \in \hat{R}^{\prime}, i_{G, M^{\prime}}\left(\tau_{\kappa^{\prime}}\right)=\underset{\kappa \in \hat{R}\left(\kappa^{\prime}\right)}{\bigoplus} \pi_{\kappa}$.

Proposition 1.7 (Herb [7]). Suppose $R$ is abelian and $C(\sigma) \simeq \mathbb{C}[R]$. Let $\pi$ be an irreducible constituent of $i_{G, M}(\sigma)$. Then $\pi=i_{G, M^{\prime}}(\tau)$ for a proper Levi subgroup $M^{\prime}$ and some $\tau \in \mathscr{E}_{t}\left(M^{\prime}\right)$, if and only if $\mathfrak{a}_{R} \neq \mathfrak{z}$. Moreover, $M^{\prime}$ and $\tau$ can be chosen so that $\tau$ is elliptic if and only if there is $a w_{0} \in R$ with $\mathfrak{a}_{R}=\mathfrak{a}_{w_{0}}$. 


\section{Parabolic subgroups of $U(n, n)$ and $U(n, n+1)$}

Let $E / F$ be a quadratic extension of locally compact, nondiscrete, nonarchimedean fields of characteristic zero. Let $x \mapsto \bar{x}$ be the nontrivial element of $\operatorname{Gal}(E / F)$. Choose an element $\beta \in E$ such that $E=F(\beta)$ and $\bar{\beta}=-\beta$. Let $G=U(n, n)$ or $U(n, n+1)$. We define $J_{n}, J_{n}^{\prime}$ by

$$
J_{n}=\left(\begin{array}{ll} 
& \beta I_{n} \\
-\beta I_{n} &
\end{array}\right) \text { and } J_{n}^{\prime}=\left(\begin{array}{ccc} 
& & \beta I_{n} \\
-\beta I_{n} &
\end{array}\right) \text {. }
$$

Then

$$
\begin{aligned}
U(n, n) & =\left\{g \in G L(2 n, E) \mid{ }^{t} \bar{g} J_{n} g=J_{n}\right\}, \\
U(n, n+1) & =\left\{g \in G L(2 n+1, E) \mid{ }^{t} \bar{g} J_{n}^{\prime} g=J_{n}^{\prime}\right\} .
\end{aligned}
$$

Each of these groups is quasi-split, but not split. Let $T$. be the maximal torus of diagonal elements in $G$. For notational convenience, we denote a diagonal element of $G L(k, E)$ by $\operatorname{diag}\left\{x_{1}, \ldots, x_{k}\right\}$. Then

$$
T=\left\{\operatorname{diag}\left\{\lambda_{1}, \lambda_{2}, \ldots, \lambda_{n}, \bar{\lambda}_{1}^{-1}, \ldots, \bar{\lambda}_{n}^{-1}\right\} \mid \lambda_{i} \in E^{*}\right\}
$$

if $G=U(n, n)$, and

$$
T=\left\{\operatorname{diag}\left\{\lambda_{1}, \ldots, \lambda_{n}, y, \bar{\lambda}_{1}^{-1}, \ldots, \bar{\lambda}_{n}^{-1}\right\} \mid \lambda_{i} \in E^{*}, y \bar{y}=1\right\}
$$

if $G=U(n, n+1)$. Let $A_{0}$ be the maximal $F$-split subtorus of $T$. Then $A_{0}$ is the collection of elements in $T$ with each $\lambda_{i} \in F^{*}$, and $y=1$ if $G=U(n, n+1)$. Notice that $\mathfrak{z}$, the real Lie algebra of the split component $Z$ of $G$, is $\{0\}$.

Let $\Phi\left(G, A_{0}\right)$ be the restricted roots of $G$ with respect to $A_{0}$. We choose the ordering on the roots so that the Borel subgroup is the collection of upper triangular matrices in $G$. If $G=U(n, n)$, then $\Phi\left(G, A_{0}\right)$ is of type $C_{n}$, while if $G=U(n, n+1)$, then $\Phi\left(G, A_{0}\right)$ is of type $B C_{n}$. Let $\Delta$ be the simple roots given by $\Delta=\left\{\alpha_{i}\right\}_{i=1}^{n}$, with $\alpha_{i}=e_{i}-e_{i+1}, \quad 1 \leq i \leq n-1$, $\alpha_{n}=2 e_{n}$ if $G=U(n, n)$, and $\alpha_{n}=e_{n}$ if $G=U(n, n+1)$.

The Weyl group $W\left(G / A_{0}\right)$ is isomorphic to $S_{n} \ltimes \mathbb{Z}_{2}^{n}$. Here $S_{n}$ acts by permutations on the matrix entries $\lambda_{i}, \quad 1 \leq i \leq n$. We use standard cycle notation for the elements of $S_{n}$. Thus, $(i j)$ interchanges $\lambda_{i}$ and $\lambda_{j}$. If $c_{i}$ is the nontrivial element of the $i$ th copy of $\mathbb{Z}_{2}$, then $c_{i}$ interchanges $\lambda_{i}$ and $\lambda_{i}^{-1}$. The element $c_{i}$ is called a sign change, because the action of $c_{i}$ on $\Phi\left(G, A_{0}\right)$ takes $e_{i}$ to $-e_{i}$. Any element which is a product of sign changes is also called a sign change.

Let $\theta \subset \Delta$. Suppose $\theta_{1} \cup \theta_{2} \cdots \cup \theta_{k}$ is the decomposition of $\theta$ as a disjoint union of connected components of the Dynkin diagram. We assume that if $\alpha_{n} \in \theta$, then $\alpha_{n} \in \theta_{k}$. Let $n_{i}=\left|\theta_{i}\right|+1$, unless $\alpha_{n} \in \theta$ and $i=k$, in which case we let $n_{k}=\left|\theta_{k}\right|$. Let $A_{\theta}$ be the subtorus of $A_{0}$ associated to $\theta$. Let $G=U(n, n)$. We denote a diagonal matrix which is scalar in blocks by $\operatorname{diag}\left\{x_{1} I_{m_{1}}, \ldots, x_{r} I_{m_{r}}\right\}$, where the $m_{i}$ are the appropriate dimensions. Then, if $\alpha_{n} \notin \theta$,

$$
A_{\theta}=\left\{\operatorname{diag}\left\{\lambda_{1} I_{n_{1}}, \ldots, \lambda_{k} I_{n_{k}}, \lambda_{1}^{-1} I_{n_{1}}, \ldots, \lambda_{k}^{-1} I_{n_{k}}\right\} \mid \lambda_{i} \in F^{*}\right\} .
$$


If $\alpha_{n} \in \theta$, then

$$
A_{\theta}=\left\{\operatorname{diag}\left\{\lambda_{1} I_{n_{1}}, \ldots, \lambda_{k-1} I_{n_{k-1}}, I_{2 n_{k}}, \lambda_{1}^{-1} I_{n_{1}}, \ldots, \lambda_{k-1}^{-1} I_{n_{k-1}}\right\} \mid \lambda_{i} \in F^{*}\right\} .
$$

If $G=U(n, n+1)$, then

$$
A_{\theta}=\left\{\operatorname{diag}\left\{\lambda_{1} I_{n_{1}}, \ldots, \lambda_{k} I_{n_{k}}, 1, \lambda_{1}^{-1} I_{n_{1}}, \ldots, \lambda_{k}^{-1} I_{n_{k}}\right\} \mid \lambda_{i} \in F^{*}\right\},
$$

if $\alpha_{n} \notin \theta$ and

$$
A_{\theta}=\left\{\operatorname{diag}\left\{\lambda_{1} I_{n_{1}}, \ldots, \lambda_{k-1} I_{n_{k-1}}, I_{2 n_{k}+1}, \lambda_{1}^{-1} I_{n_{1}}, \ldots, \lambda_{k-1}^{-1} I_{n_{k-1}}\right\} \mid \lambda_{i} \in F^{*}\right\}
$$

if $\alpha_{n} \in \theta$. Let $M_{\theta}$ be the centralizer of $A_{\theta}$. A straightforward matrix computation gives a proof of the following lemma.

Lemma 2.1. Let $\theta \subset \Delta$. Then there are integers $n_{1}, \ldots, n_{r}>0$ and $m \geq 0$, so that $n_{1}+\cdots+n_{r}+m=n$, and

$$
M_{\theta} \simeq G L\left(n_{1}, E\right) \times \cdots \times G L\left(n_{r}, E\right) \times G(m),
$$

where

$$
G(m)= \begin{cases}U(m, m) & \text { if } G=U(n, n), \\ U(m, m+1) & \text { if } G=U(n, n+1) .\end{cases}
$$

Note that we adopt the convention that

$$
G(0)= \begin{cases}1 & \text { if } G=U(n, n), \\ U(1) & \text { if } G=U(n, n+1) .\end{cases}
$$

Suppose we write $a \in A_{\theta}$ as $a=\left(\lambda_{1}, \ldots, \lambda_{r}\right)$, with each $\lambda_{i} \in F^{x}$. The Weyl group $W\left(G / A_{\theta}\right)$ is a subgroup of $S_{r} \ltimes \mathbb{Z}_{2}^{r}$. Namely, for $(i j) \in S_{r}, \quad(i j) \in$ $W\left(G / A_{\theta}\right)$ if and only if $n_{i}=n_{j}$. In this case

$$
(i j) \cdot\left(\lambda_{1}, \ldots, \lambda_{r}\right)=\left(\lambda_{1}, \ldots, \lambda_{i-1}, \lambda_{j}, \ldots, \lambda_{j-1}, \lambda_{i}, \ldots, \lambda_{r}\right) .
$$

For each $1 \leq i \leq r$, there is a block sign change $C_{i} \in W\left(G / A_{\theta}\right)$, such that $C_{i} \cdot\left(\lambda_{1}, \ldots, \lambda_{r}\right)=\left(\lambda_{1}, \ldots, \lambda_{i}^{-1}, \ldots, \lambda_{r}\right)$. We use the term "block sign change" because $C_{i}$ is the product of the $n_{i}$ sign changes $c_{b_{i}+1}, \ldots, c_{b_{i}+n_{i}} \in W\left(G / A_{0}\right)$, where $b_{i}=\sum_{j<i} n_{j}$.

If $g \in M_{\theta}$, we write $g=\left(g_{1}, \ldots, g_{r}, g^{\prime}\right)$, with $g_{i} \in G L\left(n_{i}, E\right)$ and $g^{\prime} \in G(m)$. If $(i j) \in W\left(G / A_{\theta}\right)$, then

$$
(i j) \cdot g=\left(g_{1}, \ldots, g_{i-1}, g_{j}, \ldots, g_{j-1}, g_{i}, \ldots, g_{r}, g^{\prime}\right) \text {. }
$$

For $1 \leq i \leq r$ we have $C_{i} \cdot g=\left(g_{1}, \ldots, g_{i-1}, \varepsilon\left(g_{i}\right), \ldots, g_{r}, g^{\prime}\right)$, where $\varepsilon$ is the automorphism of $G L\left(n_{i}, E\right)$ given by $\varepsilon\left(g_{i}\right)={ }^{t} \bar{g}_{i}^{-1}$. We fix a subset $\theta \subset \Delta$, and write $A=A_{\theta}$ and $M=M_{\theta}$. Let $\sigma \in \mathscr{E}_{2}(M)$. Then $\sigma \simeq \sigma_{1} \otimes \cdots \otimes \sigma_{r} \otimes \rho$, where $\sigma_{i} \in \mathscr{E}_{2}\left(G L\left(n_{i}, E\right)\right)$ and $\rho \in \mathscr{E}_{2}(G(m))$. By the above calculations, we see that $C_{i} \sigma \simeq \sigma_{1} \otimes \cdots \otimes \sigma_{i}^{\ell} \otimes \cdots \otimes \sigma_{r} \otimes \rho$, where $\sigma_{i}^{\ell}\left(g_{i}\right)=\sigma_{i}\left(\varepsilon\left(g_{i}\right)\right)$. If $s \in S_{r} \cap W(G / A)$, then $s \sigma=\sigma_{s(1)} \otimes \cdots \otimes \sigma_{s(r)} \otimes \rho$. Note we can write the conditions for $\sigma$ to be ramified as follows:

$$
\begin{aligned}
& C_{i} \sigma \simeq \sigma \Leftrightarrow \sigma_{i} \simeq \sigma_{i}^{e}, \\
& (i j) \sigma \simeq \sigma \Leftrightarrow \sigma_{i} \simeq \sigma_{j}, \\
& (i j) C_{i} C_{j} \sigma \simeq \sigma \Leftrightarrow \sigma_{i} \simeq \sigma_{j}^{\varepsilon} .
\end{aligned}
$$


That is, if $\sigma$ is ramified, then at least one of these conditions holds. If $1 \leq$ $i \leq j \leq r-1$, we denote the reduced root $e_{b_{i}}-e_{b_{j}+1}$ by $\alpha_{i j}$. We write $\beta_{i j}=e_{b_{i}}+e_{b_{j}+1}$. If $1 \leq i \leq n$, then we let

$$
\gamma_{i}= \begin{cases}2 e_{b_{i}} & \text { if } G=U(n, n), \\ e_{b_{i}} & \text { if } G=U(n, n+1) .\end{cases}
$$

Then $\left\{\alpha_{i j}, \beta_{i j}\right\}_{i \leq j} \cup\left\{a \gamma_{i} \mid a \gamma_{i} \in \Phi^{+}\right\}_{1 \leq i \leq r}$ constitutes a collection of positive reduced roots $\Phi(P, A)$.

\section{R-GROUPS FOR $U(n, n)$ AND $U(n, n+1)$}

For $\beta \in \Phi(P, A)$, the Levi subgroup $M_{\beta}$ is the centralizer of $(A \cap \operatorname{ker} \beta)^{0}$, and $\mu_{\beta}(\sigma)$ is the Plancherel measure associated to $i_{M_{\beta}, M}(\sigma)$. Thus, $\mu_{\beta}(\sigma)=0$ if and only if there is some $w \neq 1$ in $W\left(M_{\beta} / A\right)$ such that $w \sigma \simeq \sigma$, and $i_{M_{\beta}, M}(\sigma)$ is irreducible. We denote by $\Delta^{\prime}$ the collection of $\beta \in \Phi(P, A)$ such that $\mu_{\beta}(\sigma)=0$. Then

$$
R=\left\{w \in W(\sigma) \mid w \beta>0, \forall \beta \in \Delta^{\prime}\right\} .
$$

Lemma 3.1. Let $M$ be a standard Levi subgroup of $G$. For any $\sigma \in \mathscr{E}_{2}(M)$, the reduced root $\alpha_{i j} \in \Delta^{\prime}$ if and only if $\sigma_{i} \simeq \sigma_{j+1}$. Similarly, $\beta_{i j} \in \Delta^{\prime}$ if and only if $\sigma_{i} \simeq \sigma_{j+1}^{\varepsilon}$.

Proof. Note that

$$
M_{\alpha_{i j}} \simeq \prod_{k \neq i, j+1} G L\left(n_{k}, E\right) \times G L\left(n_{i}+n_{j+1}, E\right) \times G(m)
$$

and

$$
W\left(M_{\beta} / A\right)= \begin{cases}\langle(i(j+1))\rangle & \text { if } n_{i}=n_{j+1}, \\ \{1\} & \text { if } n_{i} \neq n_{j+1}\end{cases}
$$

Thus, the result for $\alpha_{i j}$ follows from the work of Ol'šanskii [12], Bernstein and Zelevinski [2], and Jacquet [8] (see [4, Lemma 3.4]). Note that $\beta_{i j}=C_{j+1}\left(\alpha_{i j}\right)$. Therefore, $\mu_{\beta_{i j}}(\sigma)=\mu_{\alpha_{i j}}\left(C_{j+1} \cdot \sigma\right)$. Thus, $\mu_{\beta_{i j}}(\sigma)=0$ if and only if $\sigma_{i} \simeq$ $\sigma_{j+1}^{e}$.

The following lemma is critical to our argument. Its proof is identical to a lemma of Keys [9].

Lemma 3.2. Let $w=s c \in R$, with $s \in S_{r}$ and $c \in \mathbb{Z}_{2}^{r}$. Then $s=1$.

Proof. By conjugating by a sign change we may assume that $c$ changes the sign of at most one $e_{b_{i}}$ in each orbit of $s$. Suppose that $s$ has a nontrivial cycle, which we may assume is of the form $(1, \ldots, j+1)$. Suppose $c$ changes no signs among $\left\{e_{b_{1}}, \ldots, e_{b_{j+1}}\right\}$. Since $w \in R$, we have $\sigma_{1} \simeq \sigma_{2} \simeq \cdots \simeq \sigma_{j+1}$. Therefore, by Lemma 3.1, $\alpha_{1 j} \in \Delta^{\prime}$. However, $w \alpha_{1 j}<0$, contradicting the assumption that $w \in R$. Now suppose $c$ changes the sign of $e_{b_{j+1}}$. Then $w \sigma \simeq$ $\sigma$ implies $\sigma_{1} \simeq \cdots \simeq \sigma_{j+1} \simeq \sigma_{1}^{e}$. Therefore, by Lemma 3.2, $\beta_{1 j} \in \Delta^{\prime}$ and $w \beta_{1 j}=s \cdot \alpha_{1 j}<0$. This contradicts our assumption that $w \in R$.

Definition 3.3. Let $\sigma \in \mathscr{E}_{2}(G L(k, E))$ and $\rho \in \mathscr{E}_{2}(G(m))$. We say that the condition $\mathscr{X}_{m, k, G}(\sigma \otimes \rho)$ holds if $i_{G(m+k), G L(k, E) \times G(m)}(\sigma \otimes \rho)$ is reducible. 
Note that if $G=G(n)=G(m+k)$ and $M=G L(k, E) \times G(m)$, then $M$ is the maximal proper Levi subgroup of $G$ determined by omitting the simple root

$$
\alpha= \begin{cases}e_{k}-e_{k+1} & \text { if } m>0 ; \\ 2 e_{n} & \text { if } m=0, G=U(n, n) ; \\ e_{n} & \text { if } m=0, G=U(n, n+1) .\end{cases}
$$

Thus, $\mathscr{X}_{m, k, G}(\sigma \otimes \rho)$ holds if and only if $\sigma \simeq \sigma^{\varepsilon}$ and $\mu_{\alpha}(\sigma) \neq 0$.

Remark. If $m=0$, then [5] determines this condition in terms of lifting from $U(k)$. Using the methods of [13], we hope to determine the condition $\mathscr{Z}_{G, m, k}(\sigma \otimes \rho)$ explicitly for all $m$. This will be the subject of joint work with Shahidi.

Theorem 3.4. Let $G=U(n, n)$ or $U(n, n+1)$. Let $P=M N$ be a parabolic subgroup of $G$. Suppose that $M \simeq G L\left(n_{1}, E\right) \times \cdots \times G L\left(n_{r}, E\right) \times G(m)$. Let $\sigma \in \mathscr{E}_{2}(M)$, with $\sigma \simeq \sigma_{1} \otimes \cdots \otimes \sigma_{r} \otimes \rho$. Let $d$ be the number of inequivalent $\sigma_{i}$ such that $\mathscr{X}_{m, n_{i}, G}\left(\sigma_{i} \otimes \rho\right)$ holds. Then $R \simeq \mathbb{Z}_{2}^{d}$.

Proof. By Lemma 3.2, $R \subset \mathbb{Z}_{2}^{r}$, the subgroup of sign changes in $W(G / A)$. For $w \in W(G / A)$, we let $R(w)=\{\alpha \in \Phi(P, A) \mid w \alpha<0\}$. Suppose $c=$ $C_{1} \cdots C_{j} \in R$. Since $c \in W(\sigma), \sigma_{i} \simeq \sigma_{i}^{\varepsilon}$ for $1 \leq i \leq j$. Therefore, $C_{i} \in W(\sigma)$ for $1 \leq i \leq j$. Note that $R\left(C_{i}\right) \subseteq R(c)$. Since $c \in R, R(c) \cap \Delta^{\prime}=\varnothing$. Thus, for $1 \leq i \leq j, R\left(C_{i}\right) \cap \Delta^{\prime}=\varnothing$, and hence $C_{i} \in R$. Therefore, $R$ is generated by single block sign changes $C_{i}$. Note that

$$
R\left(C_{i}\right)=\left\{\alpha_{i k}, \beta_{i k}\right\}_{i \leq k \leq r-1} \cup\left(\left\{a \gamma_{i}\right\}_{a=1,2} \cap \Phi^{+}\right) .
$$

Suppose $\sigma_{i} \simeq \sigma_{i}^{\varepsilon}$. By Lemma 3.1, $\alpha_{i k}, \beta_{i k} \in \Delta^{\prime}$ if and only if $\sigma_{i} \simeq \sigma_{k+1}$. By inspection, $a \gamma_{i} \in \Delta^{\prime}$ if and only if $\mathscr{X}_{m, n_{i}, G}\left(\sigma_{i} \otimes \rho\right)$ does not hold. Therefore, $C_{i} \in R$ if and only if $\sigma_{i} \neq \sigma_{k}$ for all $k>i$, and $\mathscr{X}_{m, n_{i}, G}\left(\sigma_{i} \otimes \rho\right)$ holds. Thus, there is one generator of $R$ for each equivalence class among the $\sigma_{i}$ such that $\mathscr{Z}_{m, n_{i}, G}\left(\sigma_{i} \otimes \rho\right)$ holds.

\section{Elliptic REPRESENTATIONS of $U(n, n)$ AND $U(n, n+1)$.}

In this section we use the arguments of [7] to describe the elliptic representations of $U(n, n)$ and $U(n, n+1)$. Notice that it is the manner in which the structure of the parabolics and $R$-groups for $U(n, n)$ (respectively $U(n, n+1)$ ) parallels those for $S p(2 n)$ (respectively $S O(2 n+1)$ ) which allows us to use these arguments.

Proposition 4.1. Let $G=U(n, n)$ or $U(n, n+1)$. Suppose $M$ is any Levi subgroup of $G, \sigma \in \mathscr{E}_{2}(M)$, and $R$ is the reducibility group associated to $i_{G, M}(\sigma)$. Then the commuting algebra $C(\sigma) \simeq \mathbb{C}[R]$.

Remark. By Theorems 1.4(b) and 3.4, this proves that every $i_{G, M}(\sigma)$ decomposes with multiplicity one.

Proof. We need to show that we can choose intertwining operators $T_{w}, w \in$ $R$, so that $T_{w}(\sigma)=w \sigma T_{w}$ and $T_{w_{1} w_{2}}=T_{w_{1}} T_{w_{2}}$. Suppose $\sigma=\sigma_{1} \otimes \cdots \otimes \sigma_{r} \otimes \rho$ and $\sigma$ acts on $V=V_{1} \otimes \cdots \otimes V_{r} \otimes V^{\prime}$. 
If $C_{i} \in W(\sigma)$, then $\sigma_{i} \simeq \sigma_{i}^{\varepsilon}$. Let $T_{i}: V_{i} \rightarrow V_{i}$ satisfy $T_{i} \sigma_{i}=\sigma_{i}^{\varepsilon} T_{i}$. Since $\varepsilon^{2}=1$, we must have $T_{i}^{2}=\lambda I_{V_{i}}$, with $\lambda \in \mathbb{C}^{*}$. Therefore, without loss of generality, we can assume that $T_{i}^{2}=I_{V_{i}}$. Now extend $T_{i}$ to $V$ by setting

$$
T_{i}^{V}\left(v_{1} \otimes \cdots \otimes v_{r} \otimes v^{\prime}\right)=v_{1} \otimes \cdots \otimes T_{i} v_{i} \otimes \cdots \otimes v_{r} \otimes v^{\prime} .
$$

Then $T_{i}^{V} \sigma=\left(C_{i} \sigma\right) T_{i}^{V}$ and $\left(T_{i}^{V}\right)^{2}=1$. Suppose $i<j$ and $C_{i}, C_{j} \in W(\sigma)$. Then $T_{i}^{V} T_{j}^{V}=T_{j}^{V} T_{i}^{V}$. Let $T_{C_{i}}=T_{i}^{V}$. If $c \in R$ and $c=C_{i_{1}} C_{i_{2}} \cdots C_{i_{j}}$, then $T_{c}=T_{C_{i_{1}}} \cdots T_{C_{i_{j}}}$ is an intertwining operator between $\sigma$ and $c \sigma$. Moreover, the commutativity of the operators $T_{C_{i}}$, and our normalization, guarantees that $T_{c c^{\prime}}=T_{c} T_{c^{\prime}}$ for all $c, c^{\prime} \in R$.

Lemma 4.2. For any $\sigma \in \mathscr{E}_{2}(M)$, there is a $w_{0} \in R$ with $\mathfrak{a}_{w_{0}}=\mathfrak{a}_{R}$. Further, $\mathfrak{a}_{R}=\{0\}$ if and only if $R \simeq \mathbb{Z}_{2}^{r}$.

Proof. By Theorem 3.4, we know $R \simeq \mathbb{Z}_{2}^{d}$ and $R \subset\left\langle C_{1}, \ldots, C_{r}\right\rangle$. We can assume that $R=\left\langle C_{1}, \ldots, C_{d}\right\rangle$. Let $w_{0}=C_{1} \cdots C_{d}$. Then, for all $w \in R$, $\mathfrak{a}_{w_{0}} \subseteq \mathfrak{a}_{w}$, and thus, $\mathfrak{a}_{w_{0}}=\mathfrak{a}_{R}$. If $w \in\left\langle C_{1}, \ldots, C_{r}\right\rangle$ and $\mathfrak{a}_{w}=\{0\}$, then $w=C_{1} \cdots C_{r}$. Therefore, $\mathfrak{a}_{R}=\{0\}$ if and only $R \simeq \mathbb{Z}_{2}^{r}$.

Theorem 4.3. Let $G=U(n, n)$ or $U(n, n+1)$. Let $M$ be a Levi component of $G$ with $M=G L\left(n_{1}, E\right) \times \cdots \times G L\left(n_{r}, E\right) \times G(m)$. Then the following are equivalent:

(a) $i_{G, M}(\sigma)$ has an elliptic constituent,

(b) all the constituents of $i_{G, M}(\sigma)$ are elliptic,

(c) $R \simeq \mathbb{Z}_{2}^{r}$

Proof. By Theorem 3.4, $R \simeq \mathbb{Z}_{2}^{d}$, and, by Proposition 4.1, $C(\sigma) \simeq \mathbb{C}[R]$. Therefore, by Theorem 1.5, (a) and (b) are equivalent, and both are equivalent to the existence of a $w_{0} \in R$ with $\mathfrak{a}_{w_{0}}=\{0\}$. Thus, by Lemma 4.2, (a) and (c) are equivalent.

The next result is now a direct consequence of Proposition 1.7.

Proposition 4.4. Let $G=U(n, n)$ or $U(n, n+1)$. Let $\pi \in \mathscr{E}_{t}(G)$. Then $\pi$ is either elliptic or $\pi=i_{G, M}(\tau)$ for some proper Levi subgroup $M$ of $G$ and some elliptic $\tau \in \mathscr{E}_{t}(M)$.

Suppose $R \simeq \mathbb{Z}_{2}^{r}$. For every $\kappa \in \hat{R}$, we let $\varepsilon(\kappa)=\kappa\left(C_{1} \cdots C_{r}\right)= \pm 1$. Let $1 \in \hat{R}$ denote the trivial character.

Proposition 4.5. Suppose $R \simeq \mathbb{Z}_{2}^{r}$ and $\kappa \in \hat{R}$. Let $\pi_{\kappa}$ be the corresponding irreducible constituent of $i_{G, M}(\sigma)$ and $\boldsymbol{\Theta}_{\kappa}$ its character. Then $\boldsymbol{\Theta}_{\kappa}^{e}=\varepsilon(\kappa) \boldsymbol{\Theta}_{1}^{e}$.

Proof. For $1 \leq i \leq r$, we let $M_{i}$ be the maximal Levi subgroup of $G$ containing $M$, such that $M \simeq G L\left(n_{i}\right) \times G\left(n-n_{i}\right)$. Let $R_{i}$ denote the $R$-group attached to $i_{M_{i}, M}(\sigma)$. Then, by Theorem $3.4, R_{i} \simeq \mathbb{Z}_{2}^{r-1}$ and is generated by $\left\{C_{j}\right\}_{j \neq i}$. Thus, by Theorem 4.3, every irreducible constituent $\tau$ of $i_{M_{i}, M}(\sigma)$ is elliptic. Let $\kappa_{i} \in \hat{R}_{i}$, and let $\hat{R}\left(\kappa_{i}\right)=\left\{\kappa_{i}^{+}, \kappa_{i}^{-}\right\}$, where $\kappa_{i}^{+}\left(C_{i}\right)=1$ and $\kappa_{i}^{-}\left(C_{i}\right)=-1$. Now, by Theorem 1.6, $i_{G, M_{i}}\left(\tau_{\kappa_{i}}\right)=\pi_{\kappa_{i}^{+}} \oplus \pi_{\kappa_{i}^{-}}$. Therefore, $\boldsymbol{\Theta}_{\kappa_{i}^{+}}^{e}=-\boldsymbol{\Theta}_{\kappa_{i}^{-}}^{e}$.

Now we proceed by induction. Let $s(\kappa)$ be the number of indices, $1 \leq i \leq$ $r$, such that $\kappa\left(C_{i}\right)=-1$. If $s(\kappa)=0$, then $\kappa=1, \varepsilon(\kappa)=1$, and the 
proposition is trivially true. Suppose it is true for $s(\kappa)=s$. Suppose $s(\kappa)=$ $s+1$. Let $i$ be an index with $\kappa\left(C_{i}\right)=-1$, and let $\kappa_{i}=\left.\kappa\right|_{R_{i}}$. Then $\kappa=\kappa_{i}^{-}$ and $s\left(\kappa_{i}^{+}\right)=s$. Therefore,

$$
\boldsymbol{\theta}_{\kappa}^{e}=\boldsymbol{\theta}_{\kappa_{i}^{-}}^{e}=-\boldsymbol{\theta}_{\kappa_{i}^{+}}^{e}=-\varepsilon\left(\kappa_{i}^{+}\right) \boldsymbol{\Theta}_{1}^{e}=(-1)^{s+1} \boldsymbol{\Theta}_{1}^{e}=\varepsilon(\kappa) \boldsymbol{\Theta}_{1}^{e} .
$$

\section{ACKNOWLEDGMENT}

I would like to thank Rebecca Herb and Freydoon Shahidi for their comments, discussions, and encouragement.

\section{REFERENCES}

1. J. Arthur, On elliptic temptered characters, Acta Math. 171 (1993), 73-138.

2. I. N. Bernstein and A. V. Zelevinsky, Induced representations of reductive p-adic groups. I, Ann. Sci. École Norm. Sup. (4) 10 (1977), 441-472.

3. D. Goldberg, $R$-groups and elliptic representations for $S L_{n}$, Pacific J. Math. 165 (1994), 77-92.

4. $\ldots$ Reducibility of induced representations for $S p(2 n)$ and $S O(n)$, Amer. J. Math. 484 (1994), 65-95.

5. __ Some results on reducibility for unitary groups and local Asai L-functions, J. Reine Angew. Math. 448 (1994), 65-95.

6. Harish-Chandra, Harmonic analysis on reductive p-adic groups, Proc. Sympos. Pure Math., vol. 26, Amer. Math. Soc., Providence, RI, 1973, pp. 167-192.

7. R. A. Herb, Elliptic representations for $S p(2 n)$ and $S O(n)$, Pacific J. Math. 161 (1993), 347-358.

8. H. Jacquet, Generic representations, Non Commutative Harmonic Analysis, Lecture Notes in Math., vol. 587, Springer-Verlag, New York, Heidelberg, and Berlin, 1977, pp. 91-101.

9. C. D. Keys, On the decomposition of reducible principal series representations of p-adic Chevalley groups, Pacific J. Math. 101 (1982), 351-388.

10. _ L-indistinguishability and R-groups for quasi split groups: unitary groups in even dimension, Ann. Sci. École Norm. Sup. (4) 20 (1987), 31-64.

11. A. W. Knapp and E. M. Stein, Irreducibility theorems for the principal series, Conference on Harmonic Analysis, Lecture Notes in Math., vol. 266, Springer-Verlag, New York, Heidelberg, and Berlin, 1972, pp. 197-214.

12. G. I. Ol'sanskiï, Intertwining operators and complementary series in the class of representations induced from parabolic subgroups of the genreal linear group over a locally compact division algebra, Math. USSR-Sb 22 (1974), 217-254.

13. F. Shahidi, The notion of norm and the representation theory of orthogonal groups, Invent. Math. (to appear).

14. __ On certain L-functions, Amer. J. Math. 103 (1981), 297-355.

15. A. J. Silberger, The Knapp-Stein dimension theorem for p-adic groups, Proc. Amer. Math. Soc. 68 (1978), 243-246; Correction, Proc. Amer. Math. Soc. 76 (1979), 169-170.

16. __ Introduction to harmonic anaysis on reductive p-adic groups, Math. Notes, vol. 23, Princeton Univ. Press, Princeton, NJ, 1979.

Department of Mathematics, Purdue University, West Lafayette, Indiana 47907

E-mail address: goldberg@math.purdue.edu 